\title{
Tradução e validação para a língua portuguesa de um instrumento de alfabetização em saúde bucal
}

\section{Translation and validation for the portuguese language of an oral health literacy instrument}

\section{Victor Abreu Assunção', Henrique Soares Luis' ${ }^{2}$, André Filipe Pires Silva ${ }^{3}$, Luis Soares Luis ${ }^{4}$}

'Escola Superior de Saúde do Instituto Politécnico de Portalegre, Portalegre, Portugal; Faculdade de Medicina Dentária da Universidade de Lisboa, Lisboa, Portugal; ciTechCare - Center for Innovative Care and Health Technology, Polytechnic Institute of Leiria, Leiria, Portugal. ORCID: 0000-0003-4836-0227. victorassuncao@ipportalegre.pt ${ }^{2}$ Autor para correspondência. Licenciatura em Higiene Oral, Faculdade de Medicina Dentária da Universidade de Lisboa, Lisboa, Portugal; ciTechCare Center for Innovative Care and Health Technology, Polytechnic Institute of Leiria, Leiria, Portugal. ORCID: 0000-0002-1092-7825. henrique.luis@fmd.ulisboa.pt

${ }^{3}$ Faculdade de Medicina Dentária da Universidade de Lisboa, Lisboa, Portugal. ORCID: 0000-0002-5476-4896. silva492000@gmail.com ${ }^{4}$ Escola Superior de Saúde, Instituto Politécnico de Leiria, Lairia, Portugal; ciTechCare - Center for Innovative Care and Health Technology, Polytechnic Institute of Leiria, Leiria, Portugal. ORCID: 0000-0003-2233-5752. luis.luis@ipleiria.pt

RESUMO I INTRODUÇÃO: A quantificação do nível de alfabetização em saúde é um aspeto fundamental para a saúde pública, uma vez que os indivíduos que apresentam níveis limitados de alfabetização em saúde possuem dificuldades significativas em comunicar com sucesso na sociedade atual. OBJETIVOS: Traduzir e validar a versão na língua portuguesa do Oral Health Literacy Instrument (OHLI) para avaliação da alfabetização em saúde bucal de adultos. MATERIAIS E MÉTODOS: A tradução e validação da versão na língua portuguesa do $\mathrm{OHLI}$ foi realizada em indivíduos com idade superior a 19 anos na região de Lisboa. Foram analisadas variáveis de caracterização da amostra e de alfabetização em saúde. $\mathrm{O}$ valor total do OHLI foi obtido pela soma das dimensões de leitura, compreensão e habilidade para contar. A consistência interna foi avaliada pelo $\alpha$ de Cronbach, a validação do constructo foi efetuada pela análise fatorial das questões de uma mesma dimensão e a aplicação do teste estatístico Kaiser-Meyer-Olkin. RESULTADOS: Dos 81 entrevistados, $76,5 \%$ eram mulheres. Obtiveram-se valores elevados de consistência interna, tanto para $\circ \mathrm{OHLI}$ $(0,8)$ como para as suas dimensões $(0,7)$. Para o teste de conhecimentos foi obtido um valor de 0,70. O valor final médio do OHLI foi de 81,21 tendo-se observado valores mais elevados nas pessoas com habilitações escolares superiores $(p<0,001)$. CONCLUSÕES: A versão na língua portuguesa do $\mathrm{OHLI}(\mathrm{P}-\mathrm{OHLI})$ é um instrumento confável e válido e pode ser aplicado em adultos de língua portuguesa, com as adaptações necessárias à identidade cultural, para avaliação da alfabetização em saúde bucal.

\begin{abstract}
INTRODUCTION: The quantification of health literacy's level is fundamental in public health, since individuals with limited levels of health literacy have significant difficulties in communicating successfully in today's society. OBJECTIVES: To translate and validate the Portuguese language version of Oral Health Literacy Instrument (OHLI) for the evaluation of oral health literacy in adults. METHODS AND MATERIALS: The translation and validation of the Portuguese language version of $\mathrm{OHLI}$ was carried out in individuals aged over 19 years in the Lisbon region. Variables characterizing the sample and health literacy were analyzed. The total $\mathrm{OHLI}$ value was obtained by adding the dimensions of reading, comprehension and numeracy. The internal consistency was evaluated by Cronbach's $\alpha$, the validation of the construct was performed by the factorial analysis of questions of the same dimension and by the application of the Kaiser-Meyer-Olkin test. RESULTS: Of the 81 interviewees, $76.5 \%$ were women. High values of internal consistency were obtained for both OHLI (0.8) and its dimensions (0.7). For the knowledge test, a value of 0.70 was obtained. The mean final value of $\mathrm{OHLI}$ was 81.21 , with higher values observed in people with higher education ( $p<0.001$ ). CONCLUSIONS: The Portuguese language version of $\mathrm{OHLI}(\mathrm{P}-\mathrm{OHLI})$ is a reliable and valid instrument and can be applied in Portuguesespeaking adults, with the necessary cultural adaptations, to evaluate oral health literacy.
\end{abstract}

KEYWORDS: Oral health. Literacy. Adult.

PALAVRAS-CHAVE: Saúde bucal. Alfabetização. Adulto. 
Introdução

A alfabetização em saúde (também referida como "letramento em saúde" e "literácia em saúde" no Brasil" ou "literacia em saúde" em português de Portugal) é considerada como sendo a capacidade que o indivíduo possui para obter, interpretar e compreender a informação básica de saúde e de serviços, de uma forma que seja promotora da saúde ${ }^{2,3}$. Pode também ser definida como sendo os conhecimentos e competências das pessoas para atender às demandas da saúde na sociedade moderna ${ }^{4}$. Essas competências individuais podem ser agrupadas em quatro domínios, são eles: (i) o conhecimento cultural e conceitual, (ii) a habilidade de ouvir e falar, (iii) a habilidade de escrever e ler, e por fim, (iv) a habilidade para contar ${ }^{5}$. Considerando estas competências e mantendo a atenção nas capacidades individuais, a alfabetização em saúde pode ser desenvolvida com a intervenção educacional, ficando, no entanto, sujeita à influência das interações entre o indivíduo e o meio que o rodeia ${ }^{5,6}{ }^{6}$, dos determinantes pessoais e dos comportamentos relacionados com a saúde?.

A quantificação do nível de alfabetização em saúde é um aspeto fundamental para a saúde pública, uma vez que os indivíduos que apresentam níveis limitados de alfabetização em saúde possuem dificuldades maiores em aspetos relacionados com a compreensão da notificação de receita (prescrições médicas), instruções relacionadas com a saúde e mesmo com a monitorização de parâmetros clíni$\cos ^{8-11}$, apresentando também maiores dificuldades de comunicação com os profissionais de saúde ${ }^{12}$. Esses indivíduos têm ainda mais hospitalizações, maiores custos com cuidados de saúde e pior estado de saúde geral'. Na saúde bucal, a existência de um instrumento de avaliação de alfabetização é relevante para melhorar a comunicação entre os indivíduos $^{13}$ e para ajudar no planejamento e implementação de programas de educação e promoção da mesma.

Existem diferentes instrumentos para quantificar a alfabetização em saúde, tais como o TOFHLA (Test of Functional Health Literacy in Adults) e a sua versão reduzida S-TOFHLA, e o REALM (Rapid Estimate of Adult Literacy in Medicine). Estes instrumentos foram desenhados para utilização em contexto clí- nico, não sendo adequados para outros contextos de informação em saúde ${ }^{14}$, apresentando por essa razão uma baixa fiabilidade para a avaliação da alfabetização em saúde bucal de grupos populacionais ${ }^{15}$. Um outro instrumento para a avaliação da alfabetização em saúde, o Newest Vital Sign (NVS) utiliza um rótulo nutricional de um sorvete, sendo realizadas seis perguntas que permitem avaliar 0 nível de alfabetização e habilidade para contar do indivíduo'. Esse instrumento foi traduzido, validado e aplicado, para a língua portuguesa em $2010^{16}$.

Os instrumentos REALM e TOFHLA foram adaptados para a avaliação da alfabetização em saúde bucal, estando a sua adaptação em estudo desde 2007. Foram desenvolvidos o REALD (Rapid Estimate of Adult Literacy in Dentistry), baseado no REALM, definido pelo reconhecimento de termos e o TOFHLiD (Test of Functional Health Literacy in Dentistry), baseado no TOFHLA, definido pela compreensão de leitura ${ }^{17,18}$. O REALD serve somente para identificar - reconhecimento inadequado de termos médicos e dentários. Não avalia a capacidade do indivíduo em compreender o significado desses mesmos termos $^{19}$. O TOFHLiD utiliza passagens de texto e afirmações relacionadas com o uso de fluoretos e o acesso a cuidados de saúde bucal para avaliação da capacidade de compreensão de informação escrita assim como de perceção de valores numéricos. A utilização do REALD e do TOFHLiD tem demonstrado que a alfabetização em saúde bucal é diferente da alfabetização em saúde geral pelo que devem ser avaliadas de forma separada.

O OHLI, Oral Health Literacy Instrument, é um teste de avaliação de alfabetização em saúde bucal desenvolvido em 2009 no Canadá. Este instrumento mostrou ser relevante para a avaliação da alfabetização em saúde bucal de adultos, não só no Canadá como também na versão em língua russa e chilena $^{20,21}$. Possui duas seções, a primeira, avalia a habilidade de compreensão de leitura e a segunda, a compreensão de informação numérica. A seção de leitura é composta por 38 itens com palavras omitidas de frases sobre a cárie dentária e a doença periodontal. A avaliação da habilidade para contar é composta por 19 itens de capacidade de acompanhamento de indicações médicas após consulta dentária e pós-extração dentária. Os autores do teste desenvolveram também, para aplicação 
com o OHLI, um teste de avaliação dos conhecimentos de saúde bucal, composto por 17 itens ${ }^{22}$.

Os instrumentos deste tipo devem ser traduzidos, adaptados e validados para outras línguas de modo a ajudar no planejamento de atividades de educação e promoção de saúde e também, para permitir avaliar a alfabetização em saúde bucal desses grupos populacionais, de forma a demostrar que os seus resultados são válidos para populações de língua portuguesa ${ }^{16}$.

objetivo do presente estudo foi o de traduzir e validar uma versão em português de Portugal do instrumento de avaliação de alfabetização em saúde bucal, que contém o OHLI, em adultos. A existência deste instrumento em língua portuguesa vai permitir a sua utilização para a avaliação da alfabetização em saúde bucal de uma população culturalmente diferente da original e assim aumentar o conhecimento científico numa área relevante para a prestação de cuidados de saúde bucal, que se encontrava limitada pela inexistência de um instrumento validado em língua portuguesa que permitisse o estudo da alfabetização em saúde bucal. A inovação decorrente da tradução e validação deste instrumento para a língua portuguesa permite ainda uma maior facilidade numa adaptação cultural e linguística para as diferentes versões da língua portuguesa utilizada em diferentes países do mundo.

\section{Materiais e Métodos}

Foi realizado um estudo para a validação da versão portuguesa, traduzida pelos autores, de um instrumento de avaliação de alfabetização em saúde bucal, na forma de questionário, que integra na sua estrutura o Oral Health Literacy Instrument (P-OHLI).

Este questionário agora traduzido está dividido em três dimensões, sendo a primeira, com 4 questões, a da caracterização do indivíduo, que permite a análise dos aspetos demográficos da amostra em estudo; a segunda dimensão, que não é parte integrante do OHLI, mas que a acompanha, permite a avaliação de conhecimentos de estruturas bucais, tratamentos dentários e meios de remoção de placa bacteriana, sendo composta por 17 itens; e a terceira dimensão que consiste no OHLI, composto por duas partes (57 itens) e que consiste numa ferramenta com a qual se fornece aos inquiridos uma série de informações, devendo estes, demonstrar as suas capacidades a nível de compreensão de leitura e habilidade para contar ${ }^{22}$.

A tradução do questionário foi realizada de acordo com o método descrito em 2002 e que consiste no processo "traduz - retraduz" (translate - translate back) efetuado em três etapas ${ }^{23}$. Na primeira etapa foi efetuada a tradução do documento, por dois tradutores, para a língua portuguesa; na segunda etapa realizou-se a verificação da tradução do documento solicitando-se a um terceiro tradutor para traduzir a versão portuguesa para inglês; e na terceira etapa, os dois primeiros tradutores compararam a versão original do documento (escrita em inglês) com a versão inglesa do terceiro tradutor, para harmonização ${ }^{23}$. A versão final em Português foi sujeita a observação por um painel de especialistas, composto por dois médicos dentistas, quatro higienistas orais e um doutorado em saúde pública, para avaliação de linguagem e conteúdo.

\section{Participantes e variáveis de estudo}

Para validar a versão traduzida, foi realizada a aplicação, por um único entrevistador, do questionário a 81 pessoas, maiores de 19 anos e residentes na região de Lisboa, o mais semelhantes possível com a população utilizada para a criação do questionário pelo autor original no Canadá. Foram incluídos no estudo os indivíduos sem problemas cognitivos aparentes e que foram capazes de responder de forma individual às questões do questionário. Todos os participantes deram o seu consentimento em participar, de forma voluntária, e foi-lhes explicado que poderiam desistir de participar quando quisessem.

De acordo com a literatura, é possível estabelecer um número mínimo de indivíduos adequado para a validação de um instrumento. Tal número encontra-se definido como uma amostra de 75 indivíduos com capacidade visual suficiente para ler $\circ$ instrumento e funções cognitivas que permitam a interação com o entrevistador ${ }^{24,25}$. As variáveis sociodemográficas estudadas foram: idade (anos cumpridos aquando do estudo), sexo (masculino ou feminino), nível de 
escolaridade (ensino primário não concluído, ensino primário concluído, ensino secundário não concluído, ensino secundário concluído, frequência universitária, bacharelato/licenciatura, mestrado/ doutoramento) e frequência de visitas aos profissionais de saúde bucal (2 a 4 vezes por ano, 1 vez por ano, menos de uma vez por ano e apenas quando necessito). As variáveis não demográficas estão relacionadas com as componentes do questionário, ou seja, os conhecimentos de estruturas bucais, tratamentos dentários e meios de remoção de placa bacteriana, e o nível de habilidade de leitura e habilidade para contar.

\section{Recolha da informação}

A aplicação do questionário, para recolha da informação, foi realizada em Lisboa na forma de um inquérito por questionário, onde o entrevistador entra em contacto pessoal com o participante, de acordo com o seguinte procedimento: primeiro prestou informação sobre o objetivo do estudo e a metodologia utilizada; Os participantes aceitam participar e preenchem o questionário demográfico; Os participantes preenchem o questionário de conhecimentos (parte 1); Os participantes preenchem a parte de compreensão de leitura (parte 2) do instrumento; Na parte de habilidade para contar (parte 3) o participante responde a questões relacionadas com um cartão com a imagem da notificação de receita, caixa de medicamento, cartão-de-visita e cartão de cuidados pós-cirúrgicos.

A pontuação final do $\mathrm{OHLI}$ foi realizada pela soma das questões corretas das diferentes dimensões que resultam na seguinte escala de valores, com um mínimo de 0 pontos e um máximo de 100 pontos: de 0 a 59 - nível de alfabetização inadequado, 60 a 74 -nível de alfabetização marginal e de 75 a 100 - nível de alfabetização adequado.

A análise estatística dos dados obtidos pelo instrumento, já traduzido e aplicado, foi efetuada pelo estudo das dimensões do instrumento, sendo fundamental a validação estatística do constructo e da consistência interna. A validação do construto do instrumento ocorreu pela análise fatorial das questões de uma mesma dimensão, pela aplicação do teste Kaiser-Meyer-Olkin. A validação de consistência interna foi efetuada pela aplicação da estatística Cronbach's alpha. Os dados obtidos na versão em língua portuguesa foram comparados com os dados da versão original e foram analisados utilizando o software estatístico SPSS para Windows (versão 20, SPSS Inc., Chicago, IL, USA). Os testes estatísticos foram realizados com um nível de significância de $5 \%$ após observação da normalidade dos dados. A estatística descritiva, nomeadamente proporções, media e desvio-padrão, foi utilizada para sumariar a informação demográfica, a frequência de visitas a profissionais de saúde bucal e diversos resultados de testes realizados. Os testes de estatística inferencial foram utilizados para o estudo da associação entre os valores da compreensão de literatura e habilidade para contar do OHLI, e também com o valor total do instrumento. Foi também estudada a comparação dos valores obtidos por sexo e avaliada a associação entre os níveis de alfabetização em saúde bucal (adequada, marginal e inadequada) com o sexo, nível de escolaridade e frequência de visitas a profissionais de saúde bucal. A regressão linear múltipla foi utilizada para avaliar a associação entre o valor total do OHLI como variável dependente e a idade, sexo, nível de escolaridade e frequência de visitas do participante a profissionais de saúde bucal como variáveis preditoras.

Este estudo recebeu o parecer favorável da Comissão de Ética para a Saúde da Faculdade de Medicina Dentária da Universidade de Lisboa, Portugal.

\section{Resultados}

Os participantes neste estudo $(n=81)$ apresentam uma idade compreendida entre os 20 e os 66 anos, com uma média de 41,5 anos, e um desvio padrão (dp) de 9,8 anos. A maioria dos participantes $(76,5 \%)$ são do sexo feminino, e $62,3 \%$ apresentavam um nível de escolaridade superior ou igual à frequência universitária. No que respeita às visitas a profissionais de saúde bucal, $39,5 \%$ dos inquiridos referem realizá-las 2 a 4 vezes por ano. Os resultados da caracterização demográfica podem ser observados na tabela 1. 
Tabela 1. Caracterização demográfica da amostra $(n=81)$

\begin{tabular}{|c|c|}
\hline & Valor total ou \% \\
\hline \multicolumn{2}{|l|}{ Idade } \\
\hline Idade média ( \pm dp) & $41,56( \pm 9,84)$ \\
\hline Mediana & 41 \\
\hline Mínimo & 20 \\
\hline Máximo & 66 \\
\hline \multicolumn{2}{|l|}{ Sexo (\%) } \\
\hline Masculino & 23,5 \\
\hline Feminino & 76,5 \\
\hline \multicolumn{2}{|l|}{ Nível de escolaridade (\%) } \\
\hline Ensino primário não concluído & 1,2 \\
\hline Ensino primário concluído & 6,2 \\
\hline Ensino secundário não concluído & 7,4 \\
\hline Ensino secundário concluído & 14,8 \\
\hline Frequência universitária & 11,1 \\
\hline Bacharelato/Licenciatura & 40,7 \\
\hline Mestrado/Doutoramento & 18,5 \\
\hline \multicolumn{2}{|c|}{ Frequência de visitas a profissionais de saúde bucal (\%) } \\
\hline 2 a 4 vezes por ano & 39,5 \\
\hline 1 vez por ano & 29,6 \\
\hline Menos de 1 vez por ano & 13,6 \\
\hline Apenas quando necessito & 17,3 \\
\hline
\end{tabular}

Relativamente à alfabetização em saúde bucal, o valor médio medido pela versão em língua portuguesa do OHLI (P-OHLI) é de 81,21 com um desvio-padrão de 9,44. As componentes do OHLI apresentam os seguintes valores médios e desvio-padrão: para a leitura e compreensão $41,41( \pm 4,9)$ e para a habilidade para contar $39,80( \pm 6,8)$. O valor médio relativo ao teste de conhecimentos foi de $80,10( \pm 14,6)$.

Os valores obtidos nas variáveis de estudo seguem uma distribuição normal, com exceção da variável idade, pelo que os testes estatísticos a utilizar foram os testes paramétricos, estando referidas as exceções.
A distribuição por sexo mostra que os homens apresentam maior nível de alfabetização em saúde bucal, com um valor médio de 82,21 quando comparado com 80,91 para as mulheres $(p=0,887)$. Quando se analisa a componente de leitura e compreensão verifica-se que as mulheres apresentam um resultado médio de 41,60 , superior ao valor médio dos homens que é de 40,79 ( $p=0,792)$. Na componente de habilidade para contar, voltam os homens a apresentar melhores resultados $(41,42)$ quando comparados com as mulheres que apresentam 39,30 como valor médio $(p=0,481)$. Para o teste de conhecimentos, as mulheres apresentam melhores resultados do que os homens, respetivamente 81,68 e 74,92 $(p=0,636)$. Os resultados totais dos parâmetros de alfabetização em saúde bucal ( $\mathrm{P}-\mathrm{OHLI}$ ) e os do teste de conhecimentos, podem ser observados detalhadamente na tabela 2 . 
Tabela 2. Valores médios do OHLI versão portuguesa e do teste de conhecimentos

\section{Média ( $\pm \mathrm{dp})$ \\ Mínimo \\ Máximo \\ IC $95 \%$}

Média na leitura e compreensão ( $\pm \mathrm{dp})$

Média na habilidade para contar $( \pm d p)$

\begin{tabular}{cc}
\hline OHLI versão portuguesa & Teste de conhecimentos \\
\hline $81,21( \pm 9,44)$ & $80,10( \pm 14,66)$ \\
47,38 & 35,29 \\
96,07 & 100 \\
$79,13-83,30$ & $76,86-83,34$ \\
$41,41( \pm 4,90)$ & $\mathrm{NA}$ \\
$39,80( \pm 6,80)$ & $\mathrm{NA}$
\end{tabular}

Os resultados respeitantes aos parâmetros do teste de conhecimentos e do P-OHLI, quando analisados considerando o nível de escolaridade e a frequência de visitas a profissionais de saúde bucal, podem ser observados na tabela 3 .

Tabela 3. Valores médios para o OHLI versão portuguesa e teste de conhecimentos considerando o nível de escolaridade e a frequência de visitas a profissionais de saúde bucal

\begin{tabular}{|c|c|c|}
\hline & OHLI versão portuguesa & Teste de conhecimentos \\
\hline \multicolumn{3}{|l|}{ Nível de escolaridade } \\
\hline Ensino primário $( \pm d p) *$ & $65,58( \pm 15,42) * * *$ & $68,62( \pm 21,90)$ \\
\hline Ensino secundário não concluído ( $\underline{\mathrm{d}} \mathrm{dp})$ & $77,20( \pm 17,72)$ & $81,37( \pm 14,12)$ \\
\hline Ensino secundário concluído ( \pm dp) & $82,03( \pm 6,35)$ & $85,78( \pm 14,50)$ \\
\hline Frequência universitária ( \pm dp) & $80,86( \pm 9,51)$ & $85,62( \pm 12,85)$ \\
\hline Bacharelato/Licenciatura ( $\pm \mathrm{dp})$ & $83,87( \pm 7,27)$ & $76,11( \pm 12,38)$ \\
\hline Mestrado/Doutoramento ( $\pm \mathrm{dp})$ & $87,20( \pm 3,24)$ & $85,10( \pm 14,38)$ \\
\hline Total ( $\pm d p)$ & $81,21( \pm 9,44)$ & $80,10( \pm 14,66)$ \\
\hline Valor de $\mathbf{p}^{* *}$ & $<0,01$ & 0,056 \\
\hline \multicolumn{3}{|c|}{ Frequência de visitas a profissionais de saúde bucal } \\
\hline 2 a 4 vezes por ano ( $\pm d p)$ & $82,41( \pm 9,57)$ & $81,25( \pm 10,39)$ \\
\hline 1 vez por ano $( \pm d p)$ & $81,10( \pm 9,83)$ & $81,12( \pm 16,63)$ \\
\hline Menos de 1 vez por ano ( \pm dp) & $80,27( \pm 8,73)$ & $83,42( \pm 12,28)$ \\
\hline Apenas quando necessito ( $\pm d p$ ) & $79,43( \pm 9,62)$ & $73,11( \pm 19,81)$ \\
\hline Total ( \pm dp) & $81,21( \pm 9,44)$ & $80,10( \pm 14,66)$ \\
\hline Valor de $p^{* *}$ & 0,777 & 0,258 \\
\hline
\end{tabular}

Destes resultados verificamos que somente o nível de escolaridade do ensino primário (incompleto e completo) é diferente, de forma estatisticamente significativa, dos outros níveis de escolaridade para - OHLI, não se verificando mais diferenças estatisticamente significativas nas variáveis estudadas.

A consistência interna do instrumento utilizado, quando analisada considerando o teste de conhecimento, a leitura e compreensão e a habilidade para contar, individualmente ou relacionadas entre si, foi verificada utilizando o alfa Cronbach, apresentando resultados elevados que refletem uma elevada consistência interna das componentes estudadas. Este fato demonstra uma boa consistência dos itens que as compõem. Assim, para o teste de conhecimentos, com 17 itens, foi obtido um valor de alpha de 0,7, para a compreensão e leitura, com 38 itens, e habilidade para contar com 19 itens, um valor de 0,7 em ambos os casos. Para o teste de conhecimentos e compreensão e leitura, com 55 itens, foi observado um valor de alfa de 0,8 . Para o teste de conhecimentos e habilidade para contar, com 36 itens, foi obtido um valor de alfa de 0,7. O teste de compreensão e leitura e habilidade para contar (OHLI) com 57 itens apresentou um valor de alfa de $0,8,0$ mesmo valor foi observado para $\circ \mathrm{OHLI}$ e teste de conhecimentos com 74 itens. 
Com o objetivo de explorar a relação entre o P-OHLI e o teste de conhecimentos, nas variáveis idade, sexo, nível de escolaridade e frequência de visitas a profissionais de saúde bucal, que funcionam como variáveis preditoras, foi realizado um teste de regressão linear múltipla, cujos resultados podem ser observados na tabela 4.

Tabela 4. Resultados da regressão linear múltipla para o OHLI versão portuguesa

\begin{tabular}{|c|c|c|c|}
\hline Variável preditora & $\beta$ & Erro padrão & Significância \\
\hline \multicolumn{4}{|l|}{ Modelo sem teste de conhecimento: } \\
\hline Idade & 0,054 & 0,106 & 0,609 \\
\hline Sexo & $-1,551$ & 2,307 & 0,503 \\
\hline Nível de escolaridade & 3,007 & 0,682 & $<0,01$ \\
\hline $\begin{array}{l}\text { Frequência de visitas a profissionais de saúde } \\
\text { bucal }\end{array}$ & $-0,796$ & 0,864 & 0,360 \\
\hline Constante & 66,543 & & \\
\hline \multicolumn{4}{|l|}{$\mathbf{R}^{2}=\mathbf{2 2 , 8} \%$} \\
\hline \multicolumn{4}{|l|}{ Modelo com teste de conhecimento: } \\
\hline Idade & 0,049 & 0,098 & 0,616 \\
\hline Sexo & $-3,02$ & 2,16 & 0,167 \\
\hline Nível de escolaridade & 2,82 & 0,631 & $<0,01$ \\
\hline $\begin{array}{l}\text { Frequência de visitas a profissionais de saúde } \\
\text { bucal }\end{array}$ & $-0,398$ & 0,805 & 0,622 \\
\hline Teste de conhecimento & 0,233 & 0,062 & $<0,01$ \\
\hline Constante & 51,862 & & \\
\hline $\mathbf{R}^{2}=\mathbf{3 5} \%$ & & & \\
\hline
\end{tabular}

O modelo que inclui o teste de conhecimento explica 35\% do P-OHLI, sendo este o valor mais elevado. Os valores obtidos no $\beta$ indicam a contribuição de cada uma destas variáveis para o P-OHLI. No modelo que inclui o teste de conhecimentos verifica-se que além do nível de escolaridade, o próprio teste de conhecimentos contribui de forma significativa para o resultado do P-OHLI.

A distribuição percentual do nível de alfabetização indica que a maioria dos participantes possui uma alfabetização adequada (79\%), seguido da alfabetização marginal com 18,5\% sendo, no entanto, de notar que existe uma pequena percentagem de participantes $(2,5 \%) \mathrm{com}$ um nível de alfabetização inadequado. Se verifica a existência de uma associação estatisticamente significativa entre os valores de nível de alfabetização em saúde bucal e o nível escolar, mostrando que quanto maior for o nível de escolaridade mais adequada é a alfabetização em saúde bucal. Não se observaram valores estatisticamente significativos para as associações entre o sexo e a frequência de visitas a profissionais de saúde bucal, como pode ser observado na tabela 5. 


\begin{tabular}{|c|c|c|c|}
\hline & \multicolumn{3}{|c|}{ Nível de alfabetização em saúde bucal } \\
\hline & $\begin{array}{c}\text { Adequada } \\
(\%)\end{array}$ & Marginal (\%) & Inadequado (\%) \\
\hline \multicolumn{4}{|l|}{ Sexo } \\
\hline Masculino & 84,2 & 15,8 & 0 \\
\hline Feminino & 77,4 & 19,4 & 3,2 \\
\hline Valor de $p^{*}$ & \multicolumn{3}{|c|}{0,671} \\
\hline \multicolumn{4}{|l|}{ Nível de escolaridade } \\
\hline Ensino primário & 50 & 16,7 & 33,3 \\
\hline Ensino secundário não concluído & 50 & 50 & 0 \\
\hline Ensino secundário concluído & 83,3 & 16,7 & 0 \\
\hline Frequência universiłária & 77,8 & 22,2 & 0 \\
\hline Bacharelato/Licenciatura & 78,8 & 21,2 & 0 \\
\hline Mestrado/Doutoramento & 100 & 0 & 0 \\
\hline Valor de $p^{*}$ & \multicolumn{3}{|c|}{$<0,01$} \\
\hline \multicolumn{4}{|c|}{ Frequência de visitas a profissionais de saúde bucal } \\
\hline 2 a 4 vezes por ano & 84,4 & 12,5 & 3,1 \\
\hline 1 vez por ano & 83,3 & 12,5 & 4,2 \\
\hline Menos de 1 vez por ano & 72,7 & 27,3 & 0 \\
\hline Apenas quando necessito & 64,3 & 35,7 & 0 \\
\hline Valor de $p^{*}$ & & 0,5 & \\
\hline \multicolumn{4}{|c|}{ * Valor de p obtido por um teste Qui-quadrado } \\
\hline
\end{tabular}

\section{Discussão}

A discussão dos resultados obtidos na tradução e aplicação para validação do P- OHLI é efetuada pela comparação entre os resultados obtidos na versão em língua portuguesa e na versão original do instrumento.

Esta análise indica a semelhança entre os valores obtidos nas duas versões, essencial para a confirmação da possibilidade de utilização da versão portuguesa. A versão portuguesa do OHLI foi baseada na versão original (em língua inglesa) tendo sido, antes da sua aplicação, avaliada por um painel de peritos da área. A validação foi estabelecida efetuando uma comparação entre os resultados obtidos na versão portuguesa e na versão original do $\mathrm{OHLI}$

Assim, quando observada a comparação dos valores obtidos por sexo verifica-se que estes valores apresentam bastantes semelhanças para o OHLI, não tendo sido encontradas diferenças estatisticamente significativas em ambas as versões, ( $p=0,887$ na versão portuguesa e de 0,660 na versão original).
Os resultados respeitantes aos parâmetros do teste de conhecimentos e do OHLI de ambas as versões do instrumento, quando analisados considerando o nível de escolaridade e a frequência de visitas a profissionais de saúde bucal, mostram que, e após a recodificação do nível de escolaridade, onde procedemos ao agrupamento do ensino primário não concluído com o ensino primário concluído, com a escola secundaria não concluída e a escola secundaria concluída, o que permitiu a comparação com os níveis de escolaridade da versão original. Foram também agrupados todos os graus académicos concluídos do ensino superior.

Destes resultados, verificamos que existem diferenças em ambas as versões nas classes extremas do nível de escolaridade, e isto, de forma estatisticamente significativa ( $p=0,013$ na versão portuguesa do $\mathrm{OHLI}$ e $p=0,010$ na versão original do teste de conhecimentos). Para a frequência de visitas aos profissionais de saúde bucal, foram encontradas diferenças estatisticamente significativas somente na versão original $(p=0,030)$. 
A consistência interna do instrumento utilizado, quando analisada considerando o teste de conhecimento, a leitura e compreensão e a habilidade de contar, individualmente ou relacionadas entre si, foi verificada utilizando o alfa Cronbach. Os valores obtidos são elevados, a versão original apresenta sempre valores de alfa superiores à versão portuguesa, no entanto em ambos os casos os valores são superiores a 0,7 considerado o valor ideal para um alfa de Cronbach.

A distribuição percentual do nível de alfabetização, comparado nas duas versões, mostra que na versão original não se observaram indivíduos com um nível de alfabetização inadequado, sendo que $79 \%$ dos respondentes portugueses possuem um nível de alfabetização adequado, comparando com os $89 \%$ dos respondentes da versão original. Para o nível de alfabetização marginal, os portugueses apresentaram um valor de 18,5\%, superior aos $11 \%$ dos respondentes da versão original.

Os dados obtidos permitem validar a versão do OHLI para a língua portuguesa, que se adiciona às versões existentes para a língua espanhola ${ }^{21}$ e russa $^{20}$. Comparando estas versões podemos verificar que, para a consistência interna, as três versões apresentam valores de alfa de Cronbach semelhantes, sendo de 0,812 para o instrumento em língua portuguesa, 0,887 para o instrumento em língua espanhola e de 0,895 para o instrumento em língua russa, confirmando a validade do instrumento para as diferentes línguas. $O$ valor médio do $\mathrm{OHLI}$ foi superior no teste em língua portuguesa (81.21) do que no teste em língua russa $(77,27)$.

\section{Conclusão}

Da análise dos dados obtidos na versão em língua portuguesa do instrumento de avaliação de alfabetização em saúde bucal OHLI e do teste de conhecimentos podemos concluir que esta versão se encontra adequada para aplicação na população portuguesa. Foram obtidos dados muito semelhantes, na versão em língua portuguesa, aos da versão original para os valores médios do OHLI e também para os valores médios de cada um dos seus componentes. $O$ mesmo ocorreu para os valores médios do OHLI para a distribuição por sexo dos participantes no estudo. No teste de conhecimentos, a versão em língua portuguesa apresentou resultados médios superiores à versão original, tal facto deve-se à ocorrência de um valor extremo de zero respostas certas na versão original, que influenciou a média final.

Perante os dados obtidos foi atingido o objetivo que consistia em traduzir e validar para a língua Portuguesa o $\mathrm{OHLI}$, como um instrumento de medida da alfabetização em saúde bucal, facilitando assim a sua adaptação para os países de língua portuguesa.

\section{Contribuição dos autores}

Luis HS, Assunção VA, Silva AFP e Luis LS trabalharam no desenvolvimento e implementação do projeto. Luis LS também trabalhou no texto estatístico. A seção de métodos foi desenvolvida por Assunção VA, Luis HS e Luis LS. Os quatro autores trabalharam nos resultados, discussão, conclusão e edição final. $\bigcirc$ manuscrito foi lido e aprovado por todos os autores.

\section{Conflitos de interesses}

Nenhum conflito financeiro, legal ou político envolvendo terceiros (governo, empresas e fundações privadas, etc.) foi declarado para nenhum aspecto do trabalho submetido (incluindo, mas não limitandose a subvenções e financiamentos, conselho consultivo, desenho de estudo, preparação de manuscrito, análise estatística, etc.).

\section{Referências}

1. Martins AMEBL, Almeida ER, Oliveira CC, Oliveira RCN, Pelino JEP, Santos ASF et al. Alfabetização em saúde bucal: uma revisão da literatura. Rev Assoc Paul Cir Dent. $2015 ; 69(4): 328-34$.

2. Sihota S, Lennard L. Health Literacy being able to make the most of health. London: National Consumer Council; 2004.

3. McMichael AJ, Beaglehole R. The changing global context of public health. Lancet. 2000;356(9228):495-9. doi: 10.1016/s0140-6736(00)02564-2

4. Sorensen K, Van den Broucke S, Fullam J, Doyle G, Pelikan J, Slonska $Z$ et al. Health literacy and public health: a systematic review and integration of definitions and models. BMC Public Health. 201 2; 1 2:80. doi: 10.1186/1471-2458$\underline{12-80}$ 
5. Nutbeam D, editor. Health literacy What do we know? Where do we go? Health Literacy: International Union for Health Promotion and Education Conference. Vancouver British Columbia: IUHPE; 2007.

6. Nutbeam D. The evolving concept of health literacy. Soc Sci Med. 2008;67(1 2):2072-8. doi: 10.1016/i. socscimed.2008.09.050

7. Martins $A M E B L$, Barreto SM, Santos-Neto PE, Sá MAB, Souza JGS, Haikal DS'A et al. Maior acesso à informação sobre como prevenir o câncer bucal entre idosos assistidos na atenção primária à saúde. Ciência \& Saúde Coletiva. 2015;20(7):2239-53. doi: 10.1590/1413$\underline{81232015207.15272014}$

8. Entwistle V, Williams B. Health literacy: the need to consider images as well as words. Health Expect. 2008; 11 (2):99-101. doi: $10.1111 /$ i.1369-7625.2008.00509.x

9. Weiss BD, Mays MZ, Martz W, Castro KM, DeWalt DA, Pignone MP et al. Quick assessment of literacy in primary care: the newest vital sign. Ann Fam Med. 2005;3(6):514-22. doi: $10.1370 / \mathrm{afm} .405$

10. Wills J. Health literacy: new packaging for health education or radical movement? Int J Public Health. 2009;54(1):3-4. doi: 10.1007/s00038-008-8141-7

11. Sampaio HAC, Carioca AAF, Sabry MOD, Santos PM, Coelho MAM, Passamai MPB. Letramento em saúde de diabéticos tipo 2: fatores associados e controle glicêmico. Ciência \& Saúde Coletiva. 2015;20(3):865-74. doi: $10.1590 / 1413-81232015203.12392014$

12. Pires $C$, Vigario $M$, Cavaco A. Factors influencing subjects' comprehension of a set of medicine package inserts. Int J Clin Pharm. 2016;38(4):888-98. doi: 10.1007/s1 1096-016$\underline{0305-6}$

13. Holtzman JS, Atchison KA, Macek MD, Markovic D. Oral Health Literacy and Measures of Periodontal Disease. J Periodont. 2017;88(1):78-88. doi: 10.1902/ jop.2016.160203

14. Begoray DL, Kwan B. A Canadian exploratory study to define a measure of health literacy. Health Promot Int. 2012;27(1):23-32. doi: 10.1186/1472-6831-14-141

15. Jordan JE, Osborne RH, Buchbinder R. Critical appraisal of health literacy indices revealed variable underlying constructs, narrow content and psychometric weaknesses. J Clin Epidemiol. 2011 ;64(4):366-79. doi: 10.1016/i. iclinepi.2010.04.005
16. Luis LFS. Literacia em Saúde e Alimentação Saudável: Os novos produtos e a escolha dos alimentos [tese]. Lisboa: Universidade Nova de Lisboa; 2010.

17. Gong DA, Lee JY, Rozier RG, Pahel BT, Richman JA, Vann WF Jr. Development and testing of the Test of Functional Health Literacy in Dentistry (TOFHLiD). J Public Health Dent. 2007;67(2):105-12. doi: $10.1111 /$ i.17527325.2007.00023.x

18. Lee JY, Rozier RG, Lee SY, Bender D, Ruiz RE. Development of a word recognition instrument to test health literacy in dentistry: the REALD-30--a brief communication. J Public Health Dent. 2007;67(2):94-8. doi: 10.1111/i.17527325.2007.00021.x

19. Atchison KA, Gironda MW, Messadi D, Der-Martirosian C. Screening for oral health literacy in an urban dental clinic. J Public Health Dent. 2010;70(4):269-75. doi: 10.1111/i.1752-7325.2010.00181.x

20. Blizniuk A, Ueno M, Furukawa S, Kawaguchi Y. Evaluation of a Russian version of the oral health literacy instrument (OHLI). BMC Oral Health. 2014;14:141. doi: 10.1186/1472. 6831-14-141

21. Cartes-Velasquez RA, Luengo Machuca L. Adaptation and validation of the oral health literacy instrument for the Chilean population. Int Dent J. 2017;67(4):215-20. doi: $10.1111 /$ idj.12288

22. Sabbahi DA, Lawrence HP, Limeback H, Rootman I. Development and evaluation of an oral health literacy instrument for adults. Community Dent Oral Epidemiol. 2009;37(5):451-62. doi: $10.1111 /$ i.16000528.2009.00490.x

23. Hill MM, Hill A. Investigação por questionário. Lisboa: Edições Sílabo; 2002.

24. Moreira J. Questionários: Teoria e prática. Coimbra: Edições Livraria Almedina; 2004.

25. Yurdugül H. Minimum sample size for Cronbach's coefficient alpha: a Monte-Carlo study. $\mathrm{H} \mathrm{U}$ Journal of Education. 2008;35:397-405. 